\title{
Virotherapy with newcastle disease virus for cancer treatment and its efficacy in clinical trials
}

\begin{abstract}
Cancer at now is a leading cause of death in humans worldwide due to the lack of efficient therapeutic modalities for advanced cancers. Therefore, novel therapeutic approaches have been investigated in past decades. Virotherapy is one of these approaches studied for more than 50 years with favorable results in some cancer patients. Several viruses have been used as oncolytic viruses in clinical studies. Newcastle disease virus (NDV), an avian pathogenic virus, is a promising oncolytic virus that preferentially infects and kills human cancer cells. Four major NDV-based vaccination approaches have been used in cancer patients, including vaccination with free infectious NDV particles, NDV-infected irradiated tumor cells, lysate from NDV-infected tumor cells, and dendritic cells pulsed with lysate from NDV-infected tumor cells. NDV vaccinations were well tolerated by cancer patients and reported side effects were mild. Furthermore, NDV-based vaccination can be combined with other anticancer modalities, such as other immunotherapy approaches, surgery, and chemotherapy agents, to induce stronger antitumor effects. In this article, we reviewed various aspects of NDV virotherapy in human cancers and its therapeutic efficacy in clinical trials.
\end{abstract}

Keywords: Cancer treatment, Virotherapy, Newcastle disease virus, Antitumor effects, Clinical trials
Volume 5 Issue 6 - 2017

\author{
Nemat Khansari, ${ }^{2}$ Samad Farashi Bonab! \\ 'Department of Immunology, Tehran University of medical \\ Sciences, Iran \\ ${ }^{2}$ American Medical Diagnostic Laboratory, USA
}

\begin{abstract}
Correspondence: Nemat Khansari,American Medica Diagnostic Laboratory, 1665 Garden Grove Blvd, Garden Grove CA 92843, USA, Tel 194-922-882-90,
\end{abstract}

Email nkhansari928@gmail.com

Received: July 17, 1970 | Published: July 26, 2017
Abbreviations: NDV, Newcastle Disease Virus; APMV-1, Avian Paramyxovirus Type I; IFN, Interferon; CTLA-4, Cytotoxic T Lymphocyte Associated Antigen-4; RCC, Renal Cell Carcinoma; HNSCC, Head and Neck Squamous Cell Carcinoma; IL-2, Interleukin-2; DTH, Delayed Type Hypersensitivity; Gy, Gray; BCG, Bacillus Calmette-Guerin; HN, Hemagglutinin-Neuraminidase; CD, Cluster of Differentiation; DCs, Dendritic Cells; PSA, ProstateSpecific Antigen

\section{Introduction}

In the early 1950 s, it has been shown that Newcastle disease virus (NDV) has cytotoxic effects on Ehrlich ascites tumor cells. The tumor cell death was not accompanied by demonstrable multiplication of infectious, hemagglutinating, or complements fixing particles. In addition, killing of tumor cells by NDV did not occur solely as a result of the virus reaction with the tumor cell surface. ${ }^{1,2}$ In 1964, Wheelock and Dingle reported that repeated injection of NDV to a patient with acute leukemia treated the disease. ${ }^{3}$ In 1971, Csatary reported regression of metastatic colon cancer in a Hungarian farmer following an outbreak of NDV. ${ }^{4}$ After that, naturally occurring NDV strains have been investigated as a virotherapy agent for various human cancers. NDV is an avian paramyxovirus type I (APMV-1) belonging to the genus Avulavirus in the family Paramyxoviridae. This virus infects different avian species worldwide, causing a disease known as Newcastle disease. NDV strains are classified as velogenic, mesogenic, and lentogenic according to their virulence for avian hosts. ${ }^{5} \mathrm{NDV}$ is endemic to many countries and its effects are notable in domestic poultry. ${ }^{6}$ In human, this virus can cause a transient conjunctivitis and mild flu-like symptoms. But, NDV poses no hazard to human health. So far, several mesogenic and lentogenic strains of NDV have been successfully used as an oncolytic virotherapy in mouse tumor models and cancer patients.

\section{NDV strains selectively replicate in human tumor cells}

NDV selectively replicated in human cancer cells and killed cancer cells, but not normal human cells, in vitro. In the early 1990s, it was reported that NDV, strain $73-T$, replicates in and kills human tumor cells, including fibrosarcoma, osteosarcoma, cervical carcinoma, bladder carcinoma, neuroblastoma, and Wilm's tumor cell lines, but not different normal human fibroblast cell lines, both in vitro and in vivo. Virus yield increased 10,000-fold within 24 hour in tumor and chick embryo cells supernatant, but titers remained near zero in normal fibroblast supernatant. ${ }^{7}$ Similarly, most human pancreatic tumor cell lines showed more than 700 times higher sensitivity than normal cells to the lentogenic LaSota strain of NDV killing in vitro. Normal cells were killed only by high doses of virus while most pancreatic tumor cells were killed by much lower doses. ${ }^{8}$

Several mechanisms are involved in the selective replication of NDV in tumor cells such as defects in activation of antiviral defense pathways especially type I IFN signaling pathways, ${ }^{9-11}$ activation of Rac1 expression, ${ }^{12}$ and defects in apoptotic pathways. ${ }^{13}$ Virus replication in the cytoplasm of infected cells is independent of cell proliferation. Thus, NDV is suggested as a biological agent with potential to break therapy resistance as it has the potential to replicate in non-proliferating tumor cells which are resistance to chemotherapy and radiotherapy. ${ }^{14}$ NDV can also replicate in hypoxic cancer cells. ${ }^{15}$ Induction of apoptosis and immunogenic cell death are involved in the NDV-mediated cancer cell killing. ${ }^{16,17}$

\section{NDV-based cancer virotherapy in preclinical studies}

Antineoplastic properties of NDV (strain 73 ) and the development of tumor immunity after NDV oncolysis was observed as early as $1965 .{ }^{18,19}$ In 1986, postoperative vaccination of mice with irradiated autologous tumor cells infected with NDV (lentogenic strain Ulster) 
resulted in disappearance of micrometastases from visceral organs, increased survival of vaccinated mice, and cures in about $50 \%$ of the treated mice. In contrast, no therapeutic effect was observed when non-infected autologous tumor cells were used. In addition, the therapeutic effect was less prominent when the virus was injected separately from tumor cells. ${ }^{20}$ The prevention of metastatic spread by the postoperative vaccination was paralleled by an establishment of specific systemic antitumor immunity. ${ }^{21}$ In addition, intratumoral injection of the mesogenic NDV attenuated strain PV701 (strain 73-T) resulted in complete regression of human fibrosarcoma and neuroblastoma xenografts in athymic mice. ${ }^{22}$ Intratumoral injection of recombinant NDV strains derived from the velogenic strain Italien induced syncytium formation and cell death as well as prolonged survival of tumor-bearing mice. ${ }^{23}$ Intratumoral injection of NDV in combination with systemic CTLA-4 blockade showed more therapeutic efficacy than treatment with either virotherapy or CTLA-4 blockade alone in mouse models of melanoma (B16 cells) and colon cancer (MC38 cells). ${ }^{24}$

\section{NDV as a promising virotherapy agent for cancer treatment in clinical trials}

Selective replication in tumor cells, lack of genetic recombination, and lack of interaction with host cell DNA lead to application of NDV as an attractive oncolytic virus for cancer patients. ${ }^{25}$ Furthermore, NDV virotherapy is well tolerated by cancer patients and reported side effects are mild, manifesting as flu-like symptoms and fever. Postoperatively vaccinations with NDV oncolysate (tumor cell lysate) were reported to be able to improve survival of malignant melanoma patients. ${ }^{26}$ The individuality of genetic changes in human tumor cells supports the use of autologous tumor cell vaccines. ${ }^{27}$ In the $1990 \mathrm{~s}$, NDV-infected human tumor cells were produced using tumor cell lines or tumor cells isolated from tumor samples obtained freshly from patient. Since 1990s to date, in several phase I and II clinical trials NDV-based vaccines have been used in patients with different types of cancer, including colorectal carcinoma, melanoma, renal cell carcinoma (RCC), breast cancer, ovarian cancer, glioblastoma multiform, head and neck squamous cell carcinoma (HNSCC), and prostate cancer. These viral vaccines were safe and could produce antitumor immunostimulatory responses.

\section{Vaccination with NDV particles}

In a phase II/B, multicenter clinical trial, NDV $M T H-68 / \mathrm{N}$ strain, or placebo, was administrated by inhalation for the treatment of patients with various advanced cancers. Objective clinical responses were reported in 55\% (18 patients) of NDV-vaccinated group compared to $8 \%$ ( 2 patients) in the placebo group. Significant regression of tumor(s) and/or metastases was observed in $8 \mathrm{NDV}-$ vaccinated patients, including 2 cases of complete remission. Tumor regression and/or metastases were not observed in any patients of the placebo group. Ten additional patients in the virus-vaccinated group had no further tumor progression while only two control patients showed tumor stabilization. One year survival was observed in 22 of 33 patients in the virus-vaccinated group, compared to 4 of 26 patients in the control group. There was no 2-year survivors in the control group but 7 patients in the virus-vaccinated group survived more than 2 years. ${ }^{28}$ This vaccine also produced clinical responses and long survival in 4 patients with high-graded gliomas. ${ }^{29}$ In a phase I study, multiple intravenous doses of NDV PV701 strain were administrated to patients with advanced solid cancers that were unresponsive to standard therapy $(\mathrm{n}=79)$. The most common adverse effects were flu-like symptoms that occurred mainly after the first dose and were decreased in number and severity with each subsequent dose. Tumor site-specific adverse events and acute dosing reactions were also observed. Objective responses were found at higher dose levels. Progression-free survival varied from 4-31 months. ${ }^{30}$ In colorectal patients vaccinated with LaSota strain of NDV without tumor resection $(\mathrm{n}=25), 1$-year survival rate was $96 \%$, and clinical responses include 1 patient with complete remission, 5 patients with partial remission, 16 patients with stabilized disease, and 1 patient with tumor progression. ${ }^{31}$ In three phases I clinical trials of 114 patients with solid cancers, intravenous administration of PV701 resulted in some clinical responses. General virus induced-side effects were flu-like symptoms, tumor site-specific adverse events, and infusion reactions. In the recent phase I trial of 19 patients at Hamilton, Ontario, a therapeutic regimen of desensitization, high repeat doses, and a slower infusion rate resulted in improved patient tolerability and 6 clinical responses (4 major and 2 minor) were observed. Six patients that received this regimen survived for at least 2 years. ${ }^{32}$.

\section{Vaccination with oncolysate from NDV- infected autologous tumor cells}

NDV oncolysate vaccines were used in the $1970 .{ }^{33}$ In a phase II/ III clinical trial in patients with stage III melanoma, postoperatively intracutaneous vaccinations with lysate from autologous melanoma cells infected with NDV Ulster strain in combination with administration of IL-2 did not show clinical efficacy. ${ }^{34}$

\section{Vaccination with Autologous Monocyte- Derived Dendritic Cells (DCs) pulsed with NDV-Infected tumor cell lysate}

\section{Vaccination with NDV-infected autologous tumor cells in colorectal cancer patients}

In a phase II study, postoperative vaccination of colorectal cancer patients $(\mathrm{n}=16)$ with NDV (Ulster)-infected autologous tumor cell vaccine was associated with increasing skin delayed type hypersensitivity (DTH) reactivity and a dense infiltration of predominantly helper $\mathrm{T}$ lymphocytes in the vaccination site. ${ }^{35}$ In a phase II study in patients with colorectal cancer with liver metastases $(\mathrm{n}=23)$, vaccination with $\mathrm{NDV}($ Ulster $)$-infected, irradiated (200 Gy) autologous tumor cells following curative liver resection was associated with increased sensibilisation against autologous tumor cells, measured by skin DTH reactivity. A clear correlation between increased skin DTH reaction against autologous tumor cells and recurrence-free interval was observed in vaccinated patients. ${ }^{36}$ After follow up of 18 months, a reduction in the rate of disease recurrence was observed in patients vaccinated postoperatively with NDVinfected autologous tumor cells when compared with the control group; $61 \%$ of vaccinated patients developed tumor recurrence in comparison to $87 \%$ of patients who were treated only surgically. ${ }^{37}$ In a phase II study in resected colorectal carcinoma, vaccination with NDV (Ulster)-infected autologous colorectal tumor cells $(n=48)$ resulted in better antitumor immune response than vaccination with tumor cells admixed with bacillus Calmette-Guerin (BCG) $(n=9)$. NDV-infected tumor cell vaccine induced mild side effects while vaccination with BCG-admixed tumor cells resulted in long-lasting ulcers and serious side effects. Two-year survival rate was $97.9 \%$ in the NDV-infected tumor cell vaccinated group and $66.7 \%$ in the BCG-admixed tumor cell vaccinated group. ${ }^{38}$ In colorectal patients vaccinated postoperatively with autologous tumor cell vaccine and NDV vaccine $(\mathrm{n}=310), 5,6$, and 7 -year survival rates were $66.51 \%$, $60.52 \%$, and $56.50 \%$, respectively, and in patients treated with surgery plus radiotherapy or chemotherapy $(n=257)$ they were $45.57 \%$, 
$44.76 \%$, and $43.42 \%$, respectively. There was an association between DTH reaction and the prognosis of treated patients. ${ }^{31}$

Results of a prospective large scale randomized phase II/III trial showed that NDV(Ulster)-infected autologous tumor cells fail to improve overall survival of stage IV colorectal cancer patients following resection of liver metastasis $(n=25)$ when compared with a similar number of nonvaccinated comparable patients for a followup period of about 10 years. However, metastases-free survival and overall survival in vaccinated colon cancer patients, but not in vaccinated rectal cancer patients, was more than control patients. In the control group $78.6 \%$ of patients and in the vaccinated group $30.8 \%$ of patients had died. ${ }^{39}$ In a recent phase I clinical study, vaccination of late-stage metastasized colorectal carcinoma patients $(n=14)$ with NDV-infected tumor cells attached with NDV-specific single chain antibodies with specificity for the hemagglutinin-neuraminidase $(\mathrm{HN})$ and $\mathrm{CD} 28$ (bispecific anti-HN-anti-CD28 fusion protein) induced tumor-specific $\mathrm{T}$ cells in all vaccinated patients, and 4 patients showed a partial response. ${ }^{40}$

\section{Vaccination with NDV-infected autologous tumor cells in RCC patients}

In a phase II clinical trial in patients with advanced renal cell carcinoma with distant metastases, patients after nephrectomy received multiple vaccinations with NDV (Ulster)-infected autologous tumor cells, with subsequent subcutaneous administration of low doses of IL -2 and IFN- $\alpha$. Of 40 vaccinated patients, 5 patients showed a complete response, 6 patients showed partial response, 12 patients showed stable disease (median 25 months), and 17 patients displayed tumor progression. ${ }^{41}$

\section{Vaccination with NDV-infected autologous tumor cells in breast cancer and ovarian cancer patients}

Sixty-three patients with primary breast cancer, 27 patients with metastatic pretreated breast cancer, and 31 patients with metastatic pretreated ovarian cancer were vaccinated with NDV-infected autologous tumor cell vaccines. Analysis of cohorts of patients with primary breast cancer indicated a patient's survival benefit for vaccinated patients. The survival benefit could not be attributed to vaccine alone in other patients. ${ }^{42}$

\section{Vaccination with NDV-infected autologous tumor cells in glioblastoma multiform patients}

In a nonrandomized phase II study in patients with glioblastoma multiforme postoperatively vaccinated with NDV (Ulster)-infected autologous tumor cells $(\mathrm{n}=23)$, significant increases of skin antitumor DTH reactivity and improved survival were observed in vaccinated patients when compared with non vaccinated controls $(n=87)$. Median progression-free survival and median overall survival in the vaccinated group were 40 weeks and 100 weeks, respectively, and in the non vaccinated group they were 26 weeks and 49 weeks, respectively. Increased numbers of tumor reactive memory $\mathrm{T}$ cells in the peripheral blood and CD8+ tumor infiltrating T cells were found in secondary tumors of vaccinated patients. In addition, there was one complete remission of non-resectable tumor. ${ }^{43}$

\section{Vaccination with NDV-infected autologous tumor cells in HNSCC patients}

In a nonrandomized pilot study in 20 patients with HNSCC, significant increases of antitumor skin DTH reactivity and the presence of tumor reactive $\mathrm{T}$ cells in the peripheral blood, even 5 to
7 years after vaccination, were observed in a significant proportion of patients vaccinated postoperatively with NDV-infected autologous tumor cells. ${ }^{44}$ Preconditioning of HNSCC patients with IL-2 prior to vaccination was associated with increased number of $\mathrm{T}$ cells and augmented antitumor DTH reactivity. ${ }^{45}$

\section{Vaccination with Autologous Monocyte- Derived Dendritic Cells (DCs) pulsed with NDV-infected tumor cell lysate}

\section{Vaccination with DCs pulsed with NDV-infected tumor cell lysate in a prostate cancer patient}

Complete remission of prostate cancer with extensive bone metastases has been recently reported in a patient with hormonerefractory metastatic prostate cancer who had failed standard cancer therapy. This patient received postoperatively a NDV-based combined therapy at the Immunological and Oncological Center (Cologne, Germany). This combinational therapy includes local hyperthermia, intravenous administration of NDV (MTH-68 strain), and vaccination with autologous monocyte-derived DCs pulsed with NDV-infected tumor cell lysate simultaneously with administration of IFN- $\gamma$. This postoperative combinational immunotherapy has been resulted in long-lasting dramatic decrease in prostate-specific antigen (PSA) levels, induction of antitumor memory $\mathrm{T}$ cell response, and reduction in bone metastases. ${ }^{46}$

\section{Vaccination with DCs pulsed with NDV-infected tumor cell lysate in a breast cancer patient}

At the Immunological and Oncological Center (Cologne, Germany), long-term survival of another patient with invasive ductal breast cancer and primary liver metastases was observed upon applying postoperatively radiofrequency hyperthermia of the liver, intravenous administration of NDV, and vaccinations with autologous monocyte-derived DCs pulsed with lysate from NDV-infected breast cancer cells. Sustained tumor-specific memory T cell response was detected upon DC vaccinations. ${ }^{47}$

\section{Obstacles of virotherapy}

Elicitation of neutralizing antibodies during repeated NDV vaccinations may interfere with antitumor effectiveness of NDV vaccines. The extracellular matrix in the tumor tissue can inhibit spread of viral particles or tumor reactive immune cells. The immunosuppressive microenvironment of tumor may inhibit effectiveness of vaccination. Low/poor immunogenicity of some tumors is also a major hindrance in the induction of tumor-specific immune responses. Thus, more work is needed to overcome these obstacles and augment the therapeutic efficacy of virotherapy for advanced cancers. Using reverse genetics technology, it is possible to design recombinant NDV vaccines with better efficacy from cloned DNA. Genetic manipulation of NDV to arming the virus with genes encoding cytokines or tumoricidal molecules is being investigated to improve the antitumor effects of NDV-based vaccines. ${ }^{48,49}$

\section{Conclusion}

In several preclinical and clinical studies vaccination with NDVbased vaccines resulted in tumor regression. Four approaches have been used in clinical trials, including vaccination with free infectious NDV particles, vaccination with lysate from NDV-infected tumor cells, vaccination with intact, irradiated, autologous tumor cells infected by NDV, and vaccination with DCs pulsed with lysate from NDV-infected autologous tumor cells. In general, the therapeutic 
efficacy of NDV-infected autologous tumor cell vaccines was higher than that of viral particle vaccines and virus-infected tumor cell lysate vaccines. NDV virotherapy is well tolerated by cancer patients and reported side effects are mild. DCs pulsed with NDV-infected tumor cell lysate are being examined in some centers in order to control of residual tumor cells after surgery and to augment patient's survival. NDV-based antitumor vaccination can also be combined with other anticancer modalities, such as different immunotherapy approaches and chemotherapy agents, to induce stronger antitumor effects.

\section{Acknowledgments}

The authors received no financial assistance for the research.

\section{Conflicts of interest}

The author has no conflicts of interest.

\section{References}

1. Prince AM, Ginsberg HS. Studies on the cytotoxic effect of Newcastle disease virus (NDV) on Ehrlich ascites tumor cells. I. Characteristics of the virus-cell interaction. J Immunol. 1957;79(2):94-106.

2. Prince AM, Ginsberg HS. Studies on the cytotoxic effect of Newcastle disease virus (NDV) on Ehrlich ascites tumor cells. II. The mechanism and significance of in vitro recovery from the effect of NDV. $J$ Immunol. 1957;79(2):107-112.

3. Wheelock EF, Dingle JH. Observations on the repeated administration of viruses to a patient with acute leukemia. A preliminary report. $N$ Engl J Med. 1964;271:645-651.

4. Csatary LK.Viruses in the treatment of cancer. Lancet. 1971;2:825.

5. Miller PJ, Koch G. Newcastle disease, other avian paramyxoviruses, and avian metapneumovirus infections; Newcastle disease. In Swayne DEeic, Glisson JR, McDougald LR, Nolan LK, Suarez DL, Nair V (Eds.), Diseases of poultry. 13th (edn), Wiley-Blackwell, Hoboken, NJ, USA, 2013;pp. 89-138.

6. Alexander DJ. Newcastle disease and other avian Paramyxoviruses infections. In:Saif YM, et al. (Eds.), Iowa State University Press, Ames, 2003;USA, p. 63-99.

7. Reichard KW, Lorence RM, Cascino CJ, et al. Newcastle disease virus selectively kills human tumor cells. $J$ Surg Res. 1992;52(5):448-453.

8. Walter RJ, Attar BM, Rafiq A, et al. Newcastle disease virus LaSota strain kills human pancreatic cancer cells in vitro with high selectivity. JOP. 2012;13(1):45-53.

9. Fiola C, Peeters B, Fournier P, et al. Tumor selective replication of Newcastle disease virus:Association with defects of tumor cells in antiviral defence. Int $J$ Cancer. 2006;119(2):328-338.

10. Krishnamurthy S, Takimoto T, Scroggs RA, et al. Differentially regulated interferon response determines the outcome of Newcastle disease virus infection in normal and tumor cell lines. J Virol. 2006;80(11):5145-5155.

11. Wilden H, Fournier P, Zawatzky R, et al. Expression of RIG-I, IRF3, IFN- $\beta$ and IRF7 determines resistance or susceptibility of cells to infection by Newcastle disease virus. Int J Oncol. 2009;34(4):971-982.

12. Puhlmann J, Puehler F, Mumberg D, et al. Rac 1 is required for oncolytic NDV replication in human cancer cells and establishes a link between tumorigenesis and sensitivity to oncolytic virus. Oncogene. 2010;29(15):2205-2216.

13. Mansour M, Palese P, Zamarin D. Oncolytic specificity of Newcastle disease virus is mediated by selectively for apoptosis-resistant cells. $J$ Virol. 2011;85(12):6015-6023.

14. Schirrmacher V. Oncolytic Newcastle disease virus as a prospective anti-cancer therapy. A biologic agent with potential to break therapy resistance. Exp Opin Biol Ther. 2015;15(12):1757-1771.
15. Ch'ng WC, Stanbridge EJ, Yusoff K, et al. The oncolytic activity of Newcastle disease virus in clear cell carcinoma cells in normoxic and hypoxic conditions:The interplay between VHL and interferon- $\beta$ signaling. J Interferon Cytokine Res. 2013;33(7):346-354.

16. Ahmad U, Ahmed I, Keong YY, et al. Inhibitory and apoptosis inducing effects of Newcastle disease virus strain AF2240 on mammary carcinoma cell line. Biomed Res Int. 2015;2015:127828.

17. Koks CA, Garg AD, Ehrhardt M, et al. Newcastle disease virotherapy induces long-term survival and tumor-specific immune memory in ortheotopic glioma through the induction of immunogenic cell death. Int $J$ Cancer. 2015;136(5):E313-E325.

18. Cassel WA, Garrett RE. Newcastle disease virus as an antineoplastic agent. Cancer. 1965;18(7):863-868.

19. Cassel WA, Garrett RE. Tumor immunity after viral oncolysis. $J$ Bacteriol. 1966;92(3):792.

20. Heicappell R, Schirrmacher V, von Hoegen P, et al. Prevention of metastatic spread by postoperative immunotherapy with virally modified autologous tumor cells. I. Parameters for optimal therapeutic effects. Int J Cancer. 1986;37(4):569-577.

21. Schirrmacher V, Heicappell R. Prevention of metastatic spread by postoperative immunotherapy with virally modified autologous tumor cells. II. Establishment of specific systemic anti-tumor immunity. Clin Exp Metastasis. 1987;5(2):147-156.

22. Lorence RM, Katubig BB, Reichard KW, et al. Complete regression of human fibrosarcoma xenografts after local Newcastle disease virus therapy. Cancer Res. 1994;54(23):6017-6021.

23. Wie D, Sun N, Nan G, et al. Construction of recombinant Newcastle disease virus Italien strain for oncolytic virotherapy of tumors. Hum Gene Ther. 2012;23(7):700-710.

24. Zamarin D, Holmgaard RB, Subudhi SK, et al. Localized oncolytic virotherapy overcomes systemic tumor resistance to immune checkpoint blockade immunotherapy. Sci Transl Med. 2014;6(226):226ra232.

25. Fournier P, Schirrmacher V. Oncolytic Newcastle disease virus as cutting edge between tumor and host. Biology (Basel). 2013;2(3):936-975.

26. Cassel WA, Murray DR. A ten-year follow-up on stage II malignant melanoma patients treated postsurgically with Newcastle disease virus oncolysate. Med Oncol Tumor Pharmacother. 1992;9(4):169-171.

27. Angelova M, Charoentong P, Hackl H, et al. Characterization of the immunophenotypes and antigenomes of colorectal cancers reveals distinct tumor escape mechanisms and novel targets for immunotherapy. Genome Biol. 2015;16:64.

28. Csatary LK, Eckhard S, Bukosza I, et al. Attenuated veterinary virus vaccine for the treatment of cancer. Cancer Detect Prev. 1993;17(6):619-627.

29. Csatary LK, Gosztonyi G, Szeberenyi J, et al. MTH-68/H oncolytic viral treatment in human high-grade gliomas. J Neuro Oncol. 2004;67(1-2):83-93.

30. Pecora AL, Rizvi N, Cohen GI, et al. Phase I trial of intravenous administration of PV701, an oncolytic virus, in patients with advanced solid cancers. J Clin Oncol. 2002;20(9):2251-2266.

31. Liang W, Wang H, Sun TM, et al. Application of autologous tumor cell vaccine and NDV vaccine in treatment of tumors of digestive tract. World J Gastroenterol. 2003;9(3):495-498.

32. Lorence RM, Roberts MS, O'Neil JD, et al. Phase 1 clinical experience using intravenous administration of PV701, an oncolytic Newcastle disease virus. Curr Cancer Drug Targets. 2007;7(2):157-167.

33. Murray DR, Cassel WA, Torbin AH, et al. Viral oncolysate in the management of malignant melanoma. II. Clinical studies. Cancer. 1977;40(2):680-686. 
34. Voit C, Kron M, Schwurzer-Voit M, et al. Intradermal injection of Newcastle disease virus-modified autologous melanoma cell lysate and interleukin-2 for adjuvant treatment of melanoma patients with resectable stage III disease. J Dtsch Dermatol Ges. 2003;1(2):120-125.

35. Bohle W, Schlag P, Liebrich W, et al. Postoperative active specific immunization in colorectal cancer patients with virus-modified autologous tumor-cell vaccine. First clinical results with tumor-cell vaccines modified with live but avirulent Newcastle disease virus. Cancer. 1990;66(7):1517-1523.

36. Liebrich $\mathrm{W}$, Schlag $\mathrm{P}$, Manasterski $\mathrm{M}$, et al. In vitro and clinical characterisation of a Newcastle disease virusmodified autologous tumourcell vaccine for treatment of colorectal cancer patients. Eur J cancer. 1991;27(6):703-710.

37. Schlag P, Manasterski M, Gerneth $T$, et al. Active specific immunotherapy with Newcastle-disease-virus-modified autologous tumor cells following resection of liver metastases in colorectal cancer. First evaluation of clinical response of a phase II-trial. Cancer Immunol Immunother. 1992;35(5):325-330.

38. Ockert D, Schirrmacher V, Beck N, et al. Newcastle disease virusinfected intact autologous tumor cell vaccine for adjuvant active specific immunotherapy of resected colorectal carcinoma. Clin Cancer Res. 1996;2(1):21-28.

39. Schulze T, Kemmner W, Weitz J, et al. Efficiency of adjuvant active specific immunization with Newcastle disease virus modified tumor cells in colorectal cancer patients following resection of liver metastases:Results of a prospective randomized trial. Cancer Immunol Immunother. 2009;58(1):61-69.

40. Schirrmacher V, Schlude C, Weitz J, et al. Strong T-cell costimulation can reactivate tumor antigen-specific $\mathrm{T}$ cells in late-stage metastasized colorectal carcinoma patients:Results from a phase I clinical study. Int $J$ Oncol. 2015;46(1):71-77.
41. Pomer S, Schirrmacher V, Thiele R, et al. Tumor response and 4 year survival-data of patients with advanced renal-cell carcinoma treated with autologous tumor vaccine and sucutaneous $\mathrm{R}-\mathrm{IL}-2$ and IFN- $\alpha(2 \mathrm{~b})$. Int J Oncol. 1995;6(5):947-954.

42. Ahlert T, Sauerbrei W, Bastert G, et al. Tumor-cell number and viability as quality and efficacy parameters of autologous virus-modified cancer vaccines in patients with breast and ovarian cancer. J Clin Oncol. 1977;15(4):1354-1366.

43. Steiner $\mathrm{HH}$, Bonsanto MM, Beckhove $\mathrm{P}$, et al. Antitumor vaccination of patients with glioblastoma multiforme:A pilot study to assess feasibility, safety, and clinical benefit. J Clin Oncol. 2004;22(21):4272-4281.

44. Karcher J, Dyckhoff G, Beckhove P, et al. Antitumor vaccination in patients with head and neck squamous cell carcinomas with autologous virus-modified tumor cells. Cancer Res. 2004;64(21):8057-8061.

45. Herold-Mende C, Karcher J, Dyckhoff G, et al. Antitumor immunization of head and neck squamous cell carcinoma patients with a virus-modified autologous tumor cell vaccine. $A d v$ Otorhinolaryngol. 2005;62:173-183.

46. Schirrmacher V, Bihari AS, Stuecker W, et al. Long-term remission of prostate cancer with extensive bone metastases upon immuno and virotherapy:A case report. Oncol Lett. 2014;8(6):2403-2406.

47. Schirrmacher V, Stuecker W, Lulei M, et al. Long-term survival of a breast cancer patient with extensive liver metastases upon immune and virotherapy:A case report. Immunotherapy. 2015;7(8):855-860.

48. Molouki A, Peeters B. Rescue of recombinant Newcastle disease virus:Current cloning strategies and RNA polymerase provision systems. Arch Virol. 2017;162(1):1-12.

49. Molouki A, Peeters B. Rescue of recombinant Newcastle disease virus:A short history of how it all started. Arch Virol. 2017;162(7):1845-1854. 